

\title{
Exciton States of Dynamic DNA Double Helices: Alternating dCdG Sequences
}

\author{
E. Emanuele, K. Zakrzewska, D. Markovitsi, R. Lavery, P. Millie
}

\section{To cite this version:}

E. Emanuele, K. Zakrzewska, D. Markovitsi, R. Lavery, P. Millie. Exciton States of Dynamic DNA Double Helices: Alternating dCdG Sequences. Journal of Physical Chemistry B, 2005, 109, pp.16109 -16118. 10.1021/jp051833k . hal-00084437

\section{HAL Id: hal-00084437 https://hal.science/hal-00084437}

Submitted on 7 Jul 2006

HAL is a multi-disciplinary open access archive for the deposit and dissemination of scientific research documents, whether they are published or not. The documents may come from teaching and research institutions in France or abroad, or from public or private research centers.
L'archive ouverte pluridisciplinaire HAL, est destinée au dépôt et à la diffusion de documents scientifiques de niveau recherche, publiés ou non, émanant des établissements d'enseignement et de recherche français ou étrangers, des laboratoires publics ou privés. 


\section{Exciton states of dynamic DNA double helices: alternating dCdG} sequences

\section{Emanuela Emanuele, ${ }^{a}$ Krystyna Zakrzewska, ${ }^{\text {b }}$ Dimitra Markovitsi, ${ }^{\text {a1 }}$ Richard} Lavery $^{b}$ and Philippe Milliéa

Laboratoire Francis Perrin CEA/DSM/DRECAM/SPAM - CNRS URA 2453, CEA Saclay, 91191 Gif-sur-Yvette, France

Laboratoire de Biochimie Théorique, CNRS UPR 9080, Institut de Biologie PhysicoChimique, 13, rue Pierre et Marie Curie, 75005 Paris, France

\footnotetext{
${ }^{1}$ Corresponding author: Dimitra Markovitsi (dimitra.markovitsi@cea.fr)

${ }^{a}$ Laboratoire Francis Perrin

${ }^{\mathrm{b}}$ Laboratoire de Biochimie Théorique
} 


\begin{abstract}
The present communication deals with the excited states of the alternating DNA oligomer $(\mathrm{dCdG})_{5} \cdot(\mathrm{dCdG})_{5}$ which correspond to the UV absorption band around $260 \mathrm{~nm}$. Their properties are studied in the frame of the exciton theory, combining molecular dynamics simulations and quantum chemistry data. It is shown that the dipolar coupling undergoes important variations with the site and the helix geometry. In contrast, the energy of the monomer transitions within the double helix is not sensitive to the local environment. It is thus considered to be distributed over Gaussian curves whose maximum and width are derived from the experimental absorption spectra of nucleosides in aqueous solution. The influence of the spectral width on the excited states delocalization and the absorption spectra is much stronger than that of the oligomer plasticity. About half of the excited states are delocalized over at least two bases. Many of them result from mixing of different monomer states and extend on both strands. The trends found in the simulated spectra, when going from noninteracting monomers to the duplex, are in agreement with experimental observations. Conformational changes enhance the diversity of the states which can be populated upon excitation at a given energy. The states with larger spatial extent are located close to the maximum of the absorption spectrum.
\end{abstract}




\section{Introduction}

UV irradiation absorbed by DNA bases induces photochemical reactions leading to carcinogenic mutations ${ }^{1-2}$. The major products of those photoreactions, such as bipyrimidine dimers, cytosine hydrate or oxo-dihydroxoguanine, are now well characterized. ${ }^{3,4}$ In contrast, very little is known about the excited states responsible for their formation. Thus, the energy of the lowest excited states of the bases within the helix is sometimes assumed to be different than that determined for the unstacked monomeric chromophore in solution. Attempts have been made to determine experimentally the absorption spectrum of adenine and thymine within the synthetic double helix $(\mathrm{dA})_{\mathrm{n}} \cdot(\mathrm{dT})_{\mathrm{n}},{ }^{5}$ or the energy of the thymine triplet within native DNA. ${ }^{6,7}$ Two assumptions underlie the analysis of these experimental results: excited states are localized on single bases and their energy is not site dependent.

The commonly accepted idea regarding the localization of the DNA singlet excited states goes back to the sixties. Theoretical calculations, performed in the frame of the exciton theory, concluded that the excited states in DNA should be delocalized. ${ }^{7-9}$ At the same time, they predicted that the formation of exciton states induces large spectral shifts and a visible splitting of the absorption band around $260 \mathrm{~nm}^{8}$ Since those features are not observed in the experimental absorption spectra of DNA, which were found to closely resemble the sum of the spectra of the constituent bases, the hypothesis of localized excited states prevailed ${ }^{10}$ and guided subsequent studies dealing with the photophysics and the photochemistry of DNA. The possibility of excitation delocalization having been ruled out, any difference in the spectra or the excited state reactivity between double-stranded and monomeric nucleic acids was implicitly attributed to the influence of the local environment. Such a "solvation" effect is supposed to be uniform throughout the double helix, regardless conformation or sequence. 
In parallel with those considerations, it has been discovered that the base sequence does play a role in various processes occurring after photon absorption. This is the case, for example, with energy transfer $^{11}$ or pyrimidine dimer formation in synthetic oligonucleotides. ${ }^{12}$ It is also observed in the lesion distribution around mutational hot spots. ${ }^{13}$ Moreover, delocalization of singlet excited states is suggested by the steady-state fluorescence polarization found for oligonucleotides ${ }^{14}$ and the fluorescence excitation spectra of aminopurines incorporated in a double-stranded oligonucleotide. ${ }^{15}$ Such findings incite to a theoretical reexamination of DNA excited states, taking advantage of progress made during the past decades.

Our knowledge of the excited states of the components of DNA (bases, nucleosides, nucleotides) has improved, in particular thanks to femtosecond spectroscopy. ${ }^{16-18}$ Molecular dynamics simulations, which take into account counter-ions and water molecules, provide now the possibility to obtain a detailed description of the local environment for a multitude of conformations. ${ }^{19}$ Quantum chemistry data allow a very accurate calculation of dipolar interactions which constitute the major component of the electronic coupling in the case of allowed transitions. Moreover, the difference in the interaction energy between the permanent atomic charges of a chromophore with the surrounding molecules in its ground and excited states represents an important term in the energy changes due to the local environment, in particular when excitation induces large changes in the dipole moment. Finally, the development of computational techniques allows data extracted from different areas to be combined in order to identify factors that affect the excited state properties of complex systems and determine their footprint in the absorption spectra.

Within this context, we have undertaken a systematic investigation of the singlet 
excited states related to the UV absorption by model double-stranded oligonucleotides. ${ }^{20,21} \mathrm{We}$ are interested in the way that the local environment may affect the excitation energy localized on each base and we address the question of excitation delocalization. To this end, we perform calculations combining molecular dynamics simulations, quantum chemistry data and the exciton theory. ${ }^{22-24}$ According to the exciton theory the states of a multichromophoric system are linear combinations of the excited states of each chromophore. ${ }^{22-24}$ The properties of the exciton states are obtained by diagonalization of the Hamiltonian matrix, in which the diagonal and off-diagonal terms represent the excitation energy of the monomer transitions within the system and the electronic coupling, respectively.

Our previous studies ${ }^{20,21}$ involved helices composed of only adenine-thymine base pairs, $(\mathrm{dA})_{\mathrm{n}} \cdot(\mathrm{dT})_{\mathrm{n}}$ and $(\mathrm{dAdT})_{\mathrm{n} / 2} \cdot(\mathrm{dAdT})_{\mathrm{n} / 2}$. We showed that the local environment (base sequence, conformational changes, water molecules and counter-ions) affects only slightly the excitation energy of adenine and thymine within these double helices. In contrast, drastic changes in the excited state properties are induced by the dipolar coupling which leads to delocalization of the electronic excitation with double helices having an idealized B-DNA geometry. ${ }^{20}$ Structural fluctuations reduce the spatial extent of the excited states, but excitations still remain delocalized over several bases. ${ }^{21}$

These studies neglected the fact that the UV absorption bands of nucleic acids are quite large. In other terms, it was assumed that the energy of the electronic transitions of the monomeric chromophores is not affected by changes in their structure. A general criterion for formation of delocalized excited states is the relative magnitude of the strength of the electronic coupling compared to the spectral width. ${ }^{25}$ The dipolar coupling between neighboring bases in the DNA double helix does not exceed a few hundreds of wavenumbers, 
whereas the spectral width is about one order of magnitude larger. ${ }^{20}$ Accordingly, one would expect complete localization of the excited states. However, this effect may be compensated by the existence of more than one monomer electronic transition, with different polarizations, which can be coupled. ${ }^{26}$ This indeed occurs in the case of the $(\mathrm{dA})_{10} \cdot(\mathrm{dT})_{10} \cdot{ }^{27}$

The present communication focuses on the alternating oligomer $(\mathrm{dCdG})_{5} \cdot(\mathrm{dCdG})_{5}$. We have three objectives. First, we study the influence of the local environment on the dipolar coupling and the excitation energy of the cytosine and guanine transitions within the double helix. To this end, we build the exciton Hamiltonian matrix combining ground state, excited state and transition atomic charges, extracted from quantum chemical calculations, with ground state conformations extracted from molecular dynamics simulations. In this way, we correlate the coupling fluctuations with the site and conformational variations of structural parameters. Second, we examine how the spectral width, combined with conformational changes, affects the exciton states related to photon absorption (Franck-Condon states). We illustrate their spatial extent and we quantify the various types of delocalization (spatial, electronic, intrastrand, interstrand). Third, we focus on the absorption spectrum. We compare the spectrum of the double helix to that of non-interacting monomers and we associate the observed trend to experimental data. Finally, we establish a correspondence between the absorption spectrum and the properties of the singlet excited states providing some guidelines for experimental photophysical and photochemical studies.

\section{Methodology}

2.1. Ground state molecular dynamics. The ground state conformations of $(d C d G)_{5}(d C d G)_{5}$ used for the calculation of its excited states were extracted from molecular dynamics simulations including explicit solvent and counter-ions. In order to avoid end effects 
the oligomer used in the simulations was 12 base pairs long.

Model building and simulations were performed using the AMBER 6 program $^{28}$ and the Parm98 parameter set. ${ }^{29}$ The oligomer $(\mathrm{dCdG})_{6} \cdot(\mathrm{dCdG})_{6}$ was constructed using a standard B-DNA conformation and was neutralized with $22 \mathrm{Na}^{+}$ions (placed using electrostatic potentials) and solvated with more than 6000 TIP3P water molecules in a truncated octahedral box. Molecular dynamics simulations were performed at constant temperature (300 K) and pressure ( 1 bar) using the Berendsen algorithm. ${ }^{30}$ An integration time step of 2 fs was used and all bond lengths involving hydrogens were constrained using SHAKE. ${ }^{31}$ Long-range electrostatic interactions were treated using the particle mesh Ewald (PME) approach ${ }^{32}$ with a $9 \AA$ direct space cut-off. The non-bonded pair-list was updated heuristically and the center of mass motion was removed every $10 \mathrm{ps}$ during the simulation. Initially, the water molecules and ions were relaxed by energy minimization and allowed to equilibrate at $300 \mathrm{~K}$ around the fixed DNA for 100 ps at constant volume; the entire system was then heated from 100 to 300 $\mathrm{K}$ during $10 \mathrm{ps}$ and equilibrated during 40 ps with harmonic restraints of $5.0 \mathrm{kcal} / \mathrm{mol} / \AA^{2}$ on the solute atoms at constant volume. Subsequently, the simulation was continued at constant pressure; the restraints were gradually removed over a period of 250 ps and an unrestrained simulation followed for over $4 \mathrm{~ns}$. The coordinates were saved every 1 ps. The last nanosecond was used for the further study. 100 snapshots spaced by 10 ps were selected. In order to minimize bond length and valence angle distortions the snapshots were minimized in AMBER for 1000 cycles before being used for Poisson-Boltzmann calculations of the electrostatic energy. Non-linear solutions of the Poisson-Boltzmann equation were obtained with the DELPHI program (version 2.1). ${ }^{33}$

2.2. Diagonal terms of the Hamiltonian matrix. In our calculations, we considered 
the lowest transition of cytosine $\mathrm{S}_{0} \rightarrow \mathrm{S}_{1}$ and two close lying transitions of guanine $\mathrm{S}_{0} \rightarrow \mathrm{S}_{1}$ and $\mathrm{S}_{0} \rightarrow \mathrm{S}_{2}$.

The excitation energy $E_{m}^{i}$ corresponding to the transition $S_{0} \rightarrow S_{i}$ of a chromophore $m$ within the double helix is given by:

$$
\mathrm{E}_{\mathrm{m}}^{\mathrm{i}}=\underbrace{\left(\varepsilon_{\mathrm{m}}^{\mathrm{i}}-\varepsilon_{\mathrm{m}}^{0}\right)}_{\mathrm{I}}+\underbrace{\left(\sum_{\mathrm{n} \neq \mathrm{m}} \mathrm{E}_{\mathrm{m}, \mathrm{n}}^{\mathrm{i}, 0}+\sum_{\substack{\mathrm{n} \neq \mathrm{m}>\mathrm{m} \\ \mathrm{p} \neq \mathrm{m}}} \sum_{\mathrm{n}, \mathrm{p}} \mathrm{E}^{0,0}\right)}_{\text {II }}-\underbrace{\left(\sum_{\mathrm{n} \neq \mathrm{m}} \mathrm{E}_{\mathrm{m}, \mathrm{n}}^{0,0}+\sum_{\substack{\mathrm{n} \neq \mathrm{m}>\mathrm{n} \\ \mathrm{p} \neq \mathrm{m}}} \sum_{\mathrm{n}, \mathrm{p}} \mathrm{E}_{\mathrm{n}}^{0,0}\right)}_{\text {III }}
$$

Term (I) represents the excitation energy of free monomer $m$ from its ground to its $i^{\text {th }}$ electronic state. Term (II) corresponds to the interaction energy of the system in which monomer $m$ is in its $i^{\text {th }}$ state and all others in their respective ground states. It is computed as the electrostatic energy of the system in water by solving the non-linear Poisson-Boltzmann equation with AMBER atomic charges. In this calculation, atomic charge distributions associated with the excited states of the monomers were used. Finally, term (III) is the energy of the ground-state system, and is calculated by the Poisson-Boltzmann method as well.

Atomic charges for the excited states were constructed from $a b$ initio calculations. We described the change in the monomers' electronic wavefunction upon excitation as a set of atomic charge differences (Figure 1), computed by subtraction of the CASSCF/RESP charges on the atoms of the ground state molecule from those corresponding to the excited state. The active space choose for CASSCF/RESP calculation is 10 electrons in 10 molecular orbitals for cytosine and 14 electrons in 12 molecular orbitals for guanine (we include all the valence piorbitals and the lone pairs orbitals of the heteroatoms). The basis set used is cc-pVZ.

The charges for the excited states of cytosine and guanine were obtained by adding the corresponding charge difference to the standard AMBER ground-state charges. This should 
account for the reorganization of the electronic system of the monomers upon excitation, while retaining the generality of the AMBER force field, which is very well suited to the study of nucleic acids. ${ }^{19}$ The calculation procedure is described in detail in the appendix of reference ${ }^{21}$.

\section{Figure 1}

The values attributed to term $\varepsilon_{\mathrm{m}}^{\mathrm{i}}$ in Equation 1 are chosen in two different ways. First, $\varepsilon_{\mathrm{m}}^{\mathrm{i}}$ are given constant values: $37500 \mathrm{~cm}^{-1}$ for the $\mathrm{S}_{0} \rightarrow \mathrm{S}_{1}$ transition of cytosine; $36000 \mathrm{~cm}^{-1}$ and $37500 \mathrm{~cm}^{-1}$ for the $\mathrm{S}_{0} \rightarrow \mathrm{S}_{1}$ and $\mathrm{S}_{0} \rightarrow \mathrm{S}_{2}$ transitions of guanine, respectively. Second, $\varepsilon_{\mathrm{m}}^{\mathrm{i}}$ obey Gaussian distributions whose width (fwhm) is 3750,3600 and $3750 \mathrm{~cm}^{-1}$ for the $\mathrm{S}_{0}$ $\rightarrow \mathrm{S}_{1}$ cytosine transition and the $\mathrm{S}_{0} \rightarrow \mathrm{S}_{1}$ and $\mathrm{S}_{0} \rightarrow \mathrm{S}_{2}$ guanine transitions, respectively (Table 1). Both the maximum excitation energies and the spectral width of the Gaussian functions are derived from the experimental absorption spectra of nucleosides in aqueous solutions. ${ }^{20}$

\section{Table 1}

2.3. Off-diagonal terms. The dipolar coupling was calculated using the atomic transition charge distribution model according to which the off-diagonal terms are subjected to a dipolar development. ${ }^{34}$ The resulting molecular transition dipoles $\vec{\mu}=\left\langle\Psi_{\mathrm{m}}^{\mathrm{k}}|\overrightarrow{\mathrm{r}}| \Psi_{\mathrm{m}}^{0}\right\rangle$ are then decomposed onto the atomic orbitals of the molecule, in the framework of the INDO approximation. ${ }^{35}$ Atomic charges of the three transitions were derived from quantum chemistry calculations preformed on 1-methylcytosine and 9-methylguanine. ${ }^{20}$ They were 
rescaled so that the computed transition moments to match the experimental transition moments (Table 1). The coupling corresponding to all the pairs of different bases forming the double helix was calculated.

2.4. Eigenstate properties. The detailed formalism associated with the calculation of the eigenstates is described in reference ${ }^{20}$. Diagonalization of the exciton matrix corresponding to a given helical conformation and a given distribution of monomer excitation energies yields the $\mathrm{k}$ eigenstates of the system which are linear combinations of the wavefunctions $\left\langle\Psi_{n}\right\rangle$ corresponding to the monomer transitions $|k\rangle=\sum_{n=1}^{N} C_{k, n}\left|\Psi_{n}\right\rangle$. Since the double-helix studied contains ten cytosines, with one transition each, and ten guanines, with two transitions each, it has thirty eigenstates $<\mathrm{k}>$, whose energy increases from $<1>$ to $<30>$.

The degree of delocalization of the exciton states is usually quantified by the participation ratio $P R=1 / \mathrm{L}_{\mathrm{k}}$ which represents the number of coherently coupled chromophores. ${ }^{36,37}$ When there is more than one electronic transition per chromophore, $L_{k}$ is written as follows:

$$
\mathrm{L}_{\mathrm{k}}=\sum_{\text {monomer }}\left[\sum_{\text {states } i}\left(C_{k, m}^{i}\right)^{2}\right]^{2}
$$

The sum within the square brackets represents the contribution to the eigenstate $<\mathrm{k}>$ of different electronic states belonging to the same monomer (base), e.g. the $\mathrm{S}_{1}$ and $\mathrm{S}_{2}$ states of each guanine or the $S_{1}$ state of cytosine. 


\section{Results and Discussion}

\subsection{Local environment}

The heterogeneity in the local environment of a specific base arises from its position within the double helix and the structural fluctuations of the helix. In the present section, we examine the effect of these factors on the dipolar coupling and the excitation energy.

During the nanosecond of simulation, from which we extracted 100 snapshots, the oligomer conformation shows the global characteristics of B-DNA, with an average twist of $33^{\circ}$ and average rise of $3.34 \AA$. To illustrate the structural fluctuations of the oligomer, Figure 2 shows these parameters along the double helix, together with their standard deviations calculated over the 100 conformations. It can be seen that every step has a different average value. The curves show a dinucleotide character with both rise and twist being higher for the GC steps than for the CG steps.

Figure 2

To further illustrate the variability of the relative position of the chromophores, we have plotted in Figure 3 the two intra base pair parameters, propeller and buckle. The propeller describes contra rotation angle around the long axis of the base pair and varies from $-12.5^{\circ}$ to $7.0^{\circ}$. The buckle describes contra rotation angle around the short axis of the base pair, and ranges from $-14.6^{\circ}$ to $2.7^{\circ}$. The propeller and buckle fluctuations exhibited by a given base pair due to conformational changes are also important.

Figure 3 
Conformational changes of the DNA double helix have an important impact on the dipolar coupling. The coupling variations observed for the central part of the oligomer are shown in Figure 4. By comparing the left side plots concerning C5-G6 and G6-C7, we remark that the coupling between two given transitions $\left(S_{0} \rightarrow S_{1}\right.$ of cytosine and $S_{0} \rightarrow S_{2}$ guanine) of neighboring chromophores, located on the same strand, may differ by a factor of two. This is also true for neighboring chromophores located on different strands (compare the right side plots concerning G6-G14 and G6-G16). Moreover, for certain conformations and sites (G6G16), the coupling of the $S_{0} \rightarrow S_{1}$ with the $S_{0} \rightarrow S_{2}$ transition may be as high as that between two $S_{0} \rightarrow S_{1}$ transitions whereas for other sites (G6-C14) the two coupling values differ by at least $180 \mathrm{~cm}^{-1}$. Finally, the amplitude of fluctuations observed for the coupling involving two specific bases depends on the type of the transitions. For example, for C5 and G6, the fluctuations in the coupling between $S_{0} \rightarrow S_{1}$ transitions amount to $62 \%$, but they are limited to $18 \%$ when the $S_{0} \rightarrow S_{2}$ guanine transition is involved.

The coupling between two given transitions within a base pair varies with the position of this pair along the double helix. For example, the average coupling between the $S_{0} \rightarrow S_{1}$ transitions of C5 and G16, obtained for 100 conformations, is $122 \mathrm{~cm}^{-1}$, whereas that associated with the bases of the C3-G18 pair is only $95 \mathrm{~cm}^{-1}$. Similar site variations concern the coupling between transitions of neighboring chromophores located on either the same strand or on different strands.

Figure 5 
To further analyze the dependence of the dipolar coupling on DNA flexibility, we calculated the correlation coefficient between the conformation series of dipolar coupling shown in Figure 4 and all the structural parameters associated with the relevant bases and base pairs. To our surprise, the strongest correlation was found with the slide parameter, which describes the relative displacement of neighboring bases or base pairs along their long axis. The highest correlation coefficient (0.88) was obtained for the dipolar coupling between $\mathrm{S}_{0} \rightarrow$ $S_{1}$ transition of G6 and $S_{0} \rightarrow S_{2}$ transition of G14 and the displacement of the base pairs G6C15 and C7-G14. The corresponding plots are presented in Figure 5.

\section{Figure 5}

The effect of the local environment on the diagonal energy is represented in Figure 6 where the values obtained for the ten different sites corresponding to each monomer transition within each of the 100 conformations of the double helix, that is, a total of 1000 values, are plotted. The values found for cytosine are of opposite sign and more widely spread than those for guanine. This trend is in line with the photo-induced changes observed in the permanent dipole moments (Table 2) for the three transitions: $-1.78 \mathrm{D}$ for the $\mathrm{S}_{0} \rightarrow \mathrm{S}_{1}$ transition of cytosine, +1.67 D and +0.25 D for $\mathrm{S}_{0} \rightarrow \mathrm{S}_{1}$ and $\mathrm{S}_{0} \rightarrow \mathrm{S}_{2}$ transitions of guanine, respectively. In fact, the larger the photoinduced change in the atomic charges, the more sensitive the excitation energy will be to the local environment as far as electrostatic interactions are concerned. In this respect, the present data, together with those found previously for adenine and thymine, ${ }^{21}$ show that the lowest pyrimidine transitions are more sensitive than the purine transitions. 
Figure 6

In spite the fact that the fluctuations of the monomer excitation energies clearly depend on the type of transition, they all remain very weak $\left(<15 \mathrm{~cm}^{-1}\right)$ as compared to the spectral width of room temperature solution spectra. The same result was found for $(\mathrm{dA})_{10} \cdot(\mathrm{dT})_{10}$ and


due to electrostatic interactions with the local environment cannot account for any difference between the absorption spectra of double helices and those of non interacting monomers observed at room temperature.

\section{Table 2}

\subsection{Eigenstate properties}

The spatial extent of the $(\mathrm{dCdG})_{5} \cdot(\mathrm{dCdG})_{5}$ excited states obtained by diagonalization of the exciton matrix is determined using the participation ratio $P R$. First, we consider only the effect of conformational changes assuming than the electronic transitions are devoid of any spectral width. To this end, the term $\varepsilon_{\mathrm{m}}^{\mathrm{i}}$ in Equation 1 is given constant values (Table 1). The participation ratio calculated for each eigenstate, averaged over 100 conformations, is shown in Figure 7A. It ranges from 4 to about 12. The highest values are encountered for $<\mathrm{k}>=17$ 21. Interestingly, the same eigenstates were found to be the most delocalized in the case of the alternating oligomer $(\mathrm{dAdT})_{5} \cdot(\mathrm{dAdT})_{5}\left(\right.$ Figure 7 in reference $\left.{ }^{21}\right)$. Despite this similarity, the $P R$ values corresponding to the latter oligomer do not exceed 8.2. This is due to the plasticity of 
the double helix. For example, the transition moment of the $S_{0} \rightarrow S_{1}$ transition of thymine (3.68) is similar to that of guanine (3.31) (Table 1 in reference ${ }^{20}$ ) but the coupling in a pair of guanines is higher than the coupling in a pair of thymines located at an analogous position within (dAdT) $)_{5}$ (dAdT) $)_{5}$ duplex. Thus, the average coupling values found for the G6-G14 and G6-G16 guanine pairs are 122 and 198, respectively, whereas those obtained for the corresponding thymine pairs are only 64 and 94, respectively.

Figure 7

Next, we consider the excited states of a single conformation of $(\mathrm{dCdG})_{5} .(\mathrm{dCdG})_{5}$, associated with a corresponding set of coupling terms. The diagonal terms are obtained from equation 1 where $\varepsilon_{\mathrm{m}}^{\mathrm{i}}$ obey Gaussian distributions. Since the effect of the local environment on the monomer excitation energy was found to be negligible (Figure 6), the Gaussian distributions account for homogeneous broadening. The average $P R$ found values for three different conformations, each one averaged over 500 sets of diagonal energy are plotted in Figure 7B. We observe that the average $P R$ distribution over the thirty eigenstates is similar for the three conformations examined. The three plots in Figure 7B are quite different from that in Figure 7A. The position of the larger $P R$ values has moved to lower eigenstates. The eigenstates located at the edges of the exciton band have the smallest $P R$. Most importantly, all $P R$ values have drastically decreased, ranging between 1.1 and 1.9.

We thus conclude that diagonal disorder related to the homogeneous broadening plays a more decisive role in the spatial extent of the eigenstates of the double helix than offdiagonal disorder arising from the plasticity of the double helix. The plots in Figure 7B show 
that the eigenstates are only weakly delocalized. However, this picture results from $P R$ values averaged over 500 sets of diagonal energy. Therefore, we examine below the delocalization behavior of the duplex excited states in more detail.

Delocalization of the excitation, even weak, may manifest itself in different ways. This is illustrated in Figure 8, where the topography of three typical eigenstates, associated with a single conformation and a single set of monomer excitation energies, is shown. The contribution of the excited state $\mathrm{i}$, associated with the chromophore $\mathrm{m}$, to eigenstate $<\mathrm{k}>$ is given by the coefficient $\left(\mathrm{C}_{\mathrm{k}, \mathrm{m}}^{\mathrm{i}}\right.$ ) which is drawn upwards or downwards, depending on the strand to which m belongs. We observe that each eigenstate exhibits a specific topography. In the case of $<24>, 97 \%$ of the excitation is located on the same base pair; $75 \%$ is born by the $\mathrm{S}_{2}$ state of guanine and $22 \%$ by the $\mathrm{S}_{1}$ state of cytosine. The eigenstate $<18>$ is mainly built on the $S_{1}$ and $S_{2}$ states of a guanine, but the $S_{1}$ state of the neighboring cytosine also bears $17 \%$ of the excitation. Despite their different topography, the states $<24>$ and $<18>$ have similar participation ratios, 1.6 and 1.7, respectively. Finally, the eigenstate $<9>$ is built on only one type of monomer state, the $S_{2}$ state of guanine, and it is delocalized over several bases located on both strands. The corresponding participation ratio is 3.7 .

\section{Figure 8}

The topographies shown in Figure 8 indicate that delocalization may concern different monomer electronic states $\left(\mathrm{S}_{1}\right.$ and $\mathrm{S}_{2}$ states of the same guanine), different bases (guanines belonging to various pairs), different types of bases (guanine or cytosine) and, finally, different strands. In order to examine how the excited state population is distributed with respect to 
these types of delocalization, we consider $\left(C_{k, m}^{i}\right)^{2}$ max, that is the coefficient $\left(C_{k, m}^{i}\right)^{2}$ having the highest value among the thirty coefficients describing a given eigenstate $<\mathrm{k}>$. In Figure 9 we show the distribution of the excited state population obtained for 500 sets of monomer excitation energy, that is a total of 15000 eigenstates, as a function of $\left(\mathrm{C}_{\mathrm{k}, \mathrm{m}}^{\mathrm{i}}\right)^{2}$ max. We remark that, for $42 \%$ of the eigenstates, $\left(C_{k, m}^{i}\right)^{2}{ }_{\text {max }}$ is located within the interval $0.90-1.0$. These eigenstates are almost completely localized both electronically and spatially, since more than $90 \%$ of the excitation is concentrated on one excited state of a single base. For the remaining $58 \%$ of the excited state population, an increasing part of the excitation energy is shared with at least a second electronic transition belonging either to the same or to different bases.

\section{Figure 9}

Now, we focus on the way that minor part of the excitation, corresponding to (1 $\left(\mathrm{C}_{\mathrm{k}, \mathrm{m}}^{\mathrm{i}}\right)^{2}$ max $)$, is distributed. If $\left(\mathrm{C}_{\mathrm{k}, \mathrm{m}}^{\mathrm{i}}\right)^{2}$ max is related to a guanine (or cytosine), the probability to find the remaining excitation on a cytosine (or guanine) is given by $\frac{\sum_{\text {cytosine }}\left(\mathrm{C}_{\mathrm{k}, \mathrm{m}}^{\mathrm{i}}\right)^{2}}{1-\left(\mathrm{C}_{\mathrm{k}, \mathrm{m}}^{\mathrm{i}}\right)_{\text {max }}^{2}}$ and is equal to 0.55 . This means that $55 \%$ of the excitation shared is encountered on a different type of base. Following the same reasoning, $73 \%$ of the excitation shared is located on the complementary strand.

Although the double helix eigenstates are only weakly delocalized, their oscillator strength $f$ differs a lot with respect to that of non-interacting chromophores. This can be seen in Figure 10A where the oscillator strength associated with each one of the thirty eigenstates 
corresponding to one conformation is plotted. We observe that the $f$ values are quite dispersed. They may be one order of magnitude smaller or twice as large in comparison to those of the monomers (0.19-0.22; Table 1). If we consider many sets of diagonal energy and different conformations, we observe that the differences between the oscillator strength of the various eigenstates are strongly reduced. Nevertheless, on average, the $f$ values associated with the eigenstates located at the bottom of the exciton band are lower (ca. 0.14) than those of the eigenstates at the top of the band for which $f$ is around 0.22 (Figure 10B).

Figure 10

\subsection{Spectral properties}

The question now arises whether the weak delocalization of the excitation energy found the double helix excited states is detectable in the absorption spectra. In order to answer it, we compare the spectra of the double helix with those of non-interacting chromophores. The results are shown in Figure 11 where both calculated and experimental absorption spectra are presented on a nanometer scale, for comparison with usual experimental conditions.

The experimental spectrum of the double helix is that recorded for the synthetic polymer poly(dCdG).poly(dCdG) in a phosphate buffer at room temperature. ${ }^{2}$ It is compared with that of an equimolar mixture of nucleosides, 2'-deoxycytosine and 2'-deoxyguanosine. The molar extinction coefficient $\varepsilon$ is given per base. The $\varepsilon$ at $260 \mathrm{~nm}$ of

\footnotetext{
${ }^{2}$ The nucleotides were purchased from Sigma Aldrich and dissolved in ultrapure water. The double stranded polymer was obtained from Amersham and dissolved in a phosphate buffer $\left(\mathrm{pH}=6.8 ; 0.1 \mathrm{M} \mathrm{NaH}_{2} \mathrm{PO}_{4}, 0.1 \mathrm{M}\right.$ $\mathrm{Na}_{2} \mathrm{HPO}_{4}, 0.25 \mathrm{M} \mathrm{NaCl}$ ). Absorption spectra were recorded using a Perkin Lamba 900 spectrophotometer.
} 
poly(dCdG).poly(dCdG) is taken from reference ${ }^{38}$.

The spectrum calculated for a single conformation of $(\mathrm{dCdG})_{5} \cdot(\mathrm{dCdG})_{5}$, was constructed by plotting the oscillator strength of the thirty eigenstates obtained for each one of the 500 sets of monomer transition energy values. The spectrum profiles corresponding to different conformations are quite similar, in line with the participation ratio. The calculated spectrum of non-interacting monomers was obtained by adding the three Gaussian curves which represent the energy distribution of three monomer transitions, the area under each Gaussian being proportional to the associated oscillator strength.

Figure 11

The calculated spectra of the double helix are not expected to strictly reproduce the experimental spectra. Notably, symmetric Gaussian curves were used to simulate the monomer transitions, whereas experimental bands are asymmetric. Moreover, at short wavelengths, higher order transitions overlap with those taken into account in the simulations. For those reasons, the calculated spectrum of non-interacting chromophores is different from the experimental one. Finally, charge transfer interactions which may operate in real systems are neglected in the present calculation of the exciton states. Despite the above limitations, it is interesting to compare the spectral changes occurring when going from non interacting chromophores to the double helix.

In both plots in Figure 11, we remark following trends. The monomer and double helix spectra are located in the same spectral region; no important shifts are observed. The profiles of the double helix spectra are clearly different from those of non-interacting monomers. In 
both cases, the relative intensity of the spectrum below $260 \mathrm{~nm}$ is more important for the double helix than for non-interacting monomers and its maximum intensity is lower than that of non-interacting monomers.

The calculated spectra of $(\mathrm{dCdG})_{5}$. $(\mathrm{dCdG})_{5}$ constitute an envelop corresponding to the absorption of 30 different eigenstates. Due to both diagonal disorder, induced by the spectral width, and off-diagonal disorder, generated by the plasticity of the double helix, the energy of each eigenstate and, consequently, its footprint in the absorption spectrum are quite spread. This is illustrated in Figure 12A, where the position of the thirty eigenstates $\langle\mathrm{k}\rangle$, obtained for a single conformation and 500 sets of diagonal energy distributions, is represented in the form of linear segments, together with the absorption spectrum of the double helix. We observe that the positions of the various eigenstates overlap. Thus, excitation at a given wavelength will populate eigenstates with different indexes, corresponding to various distributions of monomer energy transitions. Their relative proportion depends on the associated oscillator strength. The diversity of the excited state population formed upon photon absorption of a given energy is further amplified if various conformations are considered. Thus, although conformational changes do not have an important influence on the average properties of the eigenstates, they enhance the variety of the excited state populated at a given energy.

If internal conversion among the various eigenstates (intraband scattering) is faster than any other relaxation process, fluorescence is expected to result only from the states located at the bottom of the exciton band. Consequently, the fluorescence spectra of the double helix should not depend on the excitation wavelength. This is precisely what is experimentally observed for poly(dCdG).poly(dCdG). ${ }^{39}$

The dispersion of the participation ratio over the absorption spectrum is shown in 
Figure 12B. We note that although the average $P R$ values do not exceed 1.9 (Figure 7 ), the $P R$ associated with some states may reach values around five. The more extended eigenstates are located close to the absorption maximum. In contrast, the eigenstates located near the spectral edges are mainly localized on single bases.

\section{Conclusions and Comments}

The main conclusions of the present theoretical study on the alternating DNA oligomer $(\mathrm{dCdG})_{5} \cdot(\mathrm{dCdG})_{5}$ are summarized as follows.

Our molecular dynamics simulations have shown that the oligomer exhibits typical BDNA geometry with important variations of intra- and inter-base pair parameters. The dipolar coupling, calculated using atomic transition charges, is highly perturbed by conformational changes. In contrast, the energy of the monomer transitions $\left(\mathrm{S}_{0} \rightarrow \mathrm{S}_{1}\right.$ for cytosine, $\mathrm{S}_{0} \rightarrow \mathrm{S}_{1}$ and $\mathrm{S}_{0} \rightarrow \mathrm{S}_{2}$ for guanine) within the double helix, calculated by combining ground state and excited state transition charges with molecular dynamics simulations, including explicit water molecules and counter-ions, is not sensitive to the local environment.

The properties of the $(\mathrm{dCdG})_{5} \cdot(\mathrm{dCdG})_{5}$ excited states, calculated in the frame of exciton theory, are less affected by off-diagonal disorder associated with the plasticity of the double helix than by diagonal disorder associated with homogeneous broadening. The latter is approximated by considering that the energy of the monomer transitions is distributed over Gaussian curves, whose maxima and widths are derived from the experimental absorption spectra of nucleosides in aqueous solution. About half of the excited states are delocalized over at least two bases. Many of them result from mixing of different monomer states and extend over both strands of the DNA double helix. 
The trends found in the simulated spectra, when going from non-interacting monomers to the double helix, are in agreement with experimental findings: although no important spectral shift is observed in the oligomer spectra, their profile changes. The states with a larger spatial extent are located close to the maximum of the absorption spectrum. The plasticity of the double helix contributes to increased heterogeneity of the absorption spectra.

The conclusions drawn in the present study for $(\mathrm{dCdG})_{5} \cdot(\mathrm{dCdG})_{5}$ regarding the influence of the local environment on the monomer excitation energy within the double helix and the dipolar coupling, are quite similar to those drawn previously in the case of oligomers composed of only adenine-thymine base pairs. ${ }^{21}$ The dominant role of homogenous broadening in the spatial extent of excited states within the double helix found here confirms our findings on $(\mathrm{dA})_{10} \cdot(\mathrm{dT})_{10} \cdot{ }^{27}$ In both cases, an important part of the excited states are delocalized over at least two bases. Consequently, all these features seem to be sequence independent properties of DNA double helices.

It is important to stress that the degree of delocalization found in the present study for the excited states of $(\mathrm{dCdG})_{5}$. (dCdG) $)_{5}$ corresponds in fact to a lower limit of spatial extent. This is due to two reasons. First, we have overestimated diagonal disorder by assuming that the homogeneous spectral width is equal to the width of the experimental spectra obtained for aqueous solutions of nucleotides. In fact, the experimental width contains both homogeneous and heterogeneous contributions which cannot be separated at the present stage of our knowledge. Second, we have underestimated the electronic coupling by considering only dipolar interactions and neglecting interchromophore orbital overlap and charge transfer interactions.

The existence of excited states delocalized, even over a few bases, is expected to affect 
energy transfer in double helices, which so far has been supposed to proceed via excitation hopping. ${ }^{5,39-41}$ Intraband scattering should rapidly lead to eigenstates located to the bottom of the band. However, the properties of the latter states, whose lifetime may reach hundreds of picoseconds or nanoseconds, ${ }^{42}$ should be altered by coupling fluctuations occurring at the same time-scale. If the present study has shed some light onto the excited states directly created upon photon absorption, further work, both experimental and theoretical, is needed in order to understand the time-dependence of the DNA excited states and their relationship to photodamage.

Acknowledgment. Financial support from the ACI "Physicochimie de la Matière Complexe” is gratefully acknowledged. We thank Dr B. Bouvier for helpful discussions and Dr J. P. Dognon for having provided us with the necessary infrastructure within his group. 
TABLE 1. Properties of the Gaussian curves representing the monomer transitions used

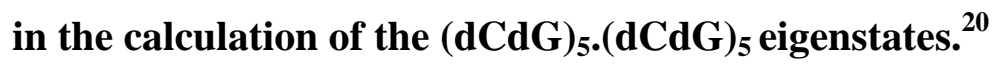

\begin{tabular}{|c|c|c|c|c|c|}
\hline \multicolumn{2}{|c|}{ Monomer transition } & $\begin{array}{c}\text { Transition } \\
\text { moment }(D)\end{array}$ & $\begin{array}{c}\text { Area } \\
(f)\end{array}$ & $\begin{array}{c}\text { Maximum } \\
\left(\mathrm{cm}^{-1}\right)\end{array}$ & $\begin{array}{c}\text { Width } \\
\left(\mathrm{fwhm} / \mathrm{cm}^{-1}\right)\end{array}$ \\
\hline Cytosine & $\mathrm{S}_{0} \rightarrow \mathrm{S}_{1}$ & 3.45 & 0.21 & 36800 & 3750 \\
\hline \multirow{2}{*}{ Guanine } & $\mathrm{S}_{0} \rightarrow \mathrm{S}_{1}$ & 3.31 & 0.19 & 36700 & 3600 \\
\cline { 2 - 6 } & $\mathrm{S}_{0} \rightarrow \mathrm{S}_{2}$ & 3.31 & 0.21 & 40300 & 3750 \\
\hline
\end{tabular}

TABLE 2: Norms of the permanent dipole moments (in Debye) of the electronic states of cytosine and guanine calculated from atomic charges

$\begin{array}{ccccc}\text { Cytosine } \mathbf{S}_{\mathbf{0}} & \text { Cytosine } \mathbf{S}_{\mathbf{1}} & \text { Guanine } \mathbf{S}_{\mathbf{0}} & \text { Guanine } \mathbf{S}_{\mathbf{1}} & \text { Guanine } \mathbf{S}_{\mathbf{2}} \\ 6.19 & 4.41 & 6.26 & 6.51 & 7.93\end{array}$




\section{Figure Captions}

1. Difference between the excited state and the ground state atomic charges corresponding to the $S_{0} \rightarrow S_{1}$ transition of cytosine and the $S_{0} \rightarrow S_{1}$ and $S_{0} \rightarrow S_{2}$ transitions of guanine; negative and positive charges appear as black and grey disks, respectively. The area of the disks is proportional to the absolute value of the change in charge.

2. Intrer-base pair parameters of $(\mathrm{dCdG})_{5} \cdot(\mathrm{dCdG})_{5}$. Twist is the rotation around the helical axis. Rise is the distance between the successive base pairs along the helical axis. The values are averaged over 100 conformations. The error bars correspond to standard deviation.

3. Intra-base pair parameters of $(\mathrm{dCdG})_{5} \cdot(\mathrm{dCdG})_{5}$. Propeller and buckle are contra-rotations around the long and short axes of the base pair, respectively. Average values over 100 conformations. The error bars correspond to standard deviation.

4. Dipolar coupling determined for 100 conformations of $(\mathrm{dCdG})_{5} \cdot(\mathrm{dCdG})_{5}$ extracted from the molecular dynamics simulations. Left side plots: coupling between transitions of neighboring cytosine and guanine pairs located in the same strand. Right side plots: coupling between transitions of two neighboring guanines located on different strands. The double strand is schematically represented by grey (cytosine) and white (guanine) circles. 
5. Dependence of dipolar coupling on the $(\mathrm{dCdG})_{5} \cdot(\mathrm{dCdG})_{5}$ conformation. Coupling between the $\mathrm{S}_{0} \rightarrow \mathrm{S}_{1}$ transitions of G6 and G14 (grey line; left axis). Slide parameter between the base pairs G6-C15 et C7-G14 (black line; right axis). Slide describes the relative displacement of the successive base pairs along their long axis.

6. Modification of the monomer excitation energy due to electrostatic interactions with the local environment. Energy distribution determined for the ten cytosines and ten guanines of $(\mathrm{dCdG})_{5} \cdot(\mathrm{dCdG})_{5}$ in 100 conformations extracted from the molecular dynamics simulations.

7. Participation ratio corresponding to the 30 eigenstates of the $(\mathrm{dCdG})_{5} \cdot(\mathrm{dCdG})_{5}$. (A) Only the local environment effect on the diagonal energy is considered (average values over 100 conformations). (B) Average values over 500 distributions of monomer transition energies; black, grey and white bars correspond to different oligomer conformations.

8. Topography of the eigenstates $\langle 24>,\langle 18>$, and $\langle 9\rangle$ obtained for a same conformation of $(\mathrm{dCdG})_{5} \cdot(\mathrm{dCdG})_{5}$ and the same diagonal energy distribution. The coefficients $\left(\mathrm{C}_{\mathrm{k}, \mathrm{m}}^{\mathrm{i}}\right)$ represent the contribution of chromophore $\mathrm{m}$ in its $\mathrm{i}^{\text {th }}$ excited state to eigenstate $<\mathrm{k}>$. The upper and lower parts of each histogram refer to chromophores located on each of the two strands. PR is the participation ratio. 
9. Distribution of the eigenstate population of $(\mathrm{dCdG})_{5} \cdot(\mathrm{dCdG})_{5}$ as a function of the maximum value encountered among the $30\left(\mathrm{C}_{\mathrm{k}, \mathrm{m}}^{\mathrm{i}}\right)^{2}$ coefficients. A single conformation and 500 diagonal energy sets are considered.

10. Oscillator strength corresponding to the 30 eigenstates of $(\mathrm{dCdG})_{5}$. $(\mathrm{dCdG})_{5}$. (A) A single conformation and a single distribution of the monomer transition energies, both chosen randomly, are considered. (B) Average values over 500 distributions of monomer transition energies obtained for a single conformation of the double helix.

11. Comparison between the absorption spectra of the double helix (solid lines) and those of non-interacting monomers (dashed lines). The experimental spectra were obtained for poly(dCdG).poly(dCdG) and an equimolar mixture of nucleosides $d C$ and $d G$; the molar extinction coefficients $(\varepsilon)$ are given per base. The calculated spectra of $(\mathrm{dCdG})_{5} \cdot(\mathrm{dCdG})_{5}$ were obtained for two different conformations (500 diagonal energy distributions per conformation); the total oscillator strength was normalized per base pair. The monomer calculated spectrum is the sum of the Gaussians corresponding to the three monomer transitions considered.

12. Position of the eigenstates (linear segments) and their participation ratio (circles) with respect to the absorption spectrum obtained for a single conformation of $(\mathrm{dCdG})_{5} .(\mathrm{dCdG})_{5}$ and 500 sets of monomer excitation energy. 


\section{REFERENCES}

(1) Cadet, J.; Vigny, P. The Photochemistry of Nucleic Acids. In Bioorganic Photochemistry; Morrison, H., Ed.; John Wiley \& Sons: New York, 1990; pp 1.

(2) Dumaz, N.; Van Kranen, H. J.; De Vries, A.; Berg, R. J. W.; Wester, P. W.; Van Kreijl, C. F.; Sarasin, A.; Daya-Grosjean, L.; De Gruijl, F. R. Carcinogenesis 1997, 18, 897.

(3) Cadet, J.; Berger, M.; Douki, T.; Morin, B.; S., R.; Ravanat, J. L.; Spinelli, S. Biol. Chem. 1997, 378, 1275.

(4) Cadet, J.; Douki, T.; Pouget, J. P.; Ravanat, J. L.; Sauvaigo, S. Curr Prob. Dermat. 2001, 29, 62.

(5) Georghiou, S.; Phillips, G. R.; Ge, G. Biopolymers 1992, 32, 1417.

(6) Gut, I. G.; Wood, P. D.; Redmond, R., W. J. Am. Chem. Soc. 1996, 118, 2366.

(7) Tinoco Jr., I. J. Am. Chem. Soc. 1960, 82, 4785.

(8) Rhodes, W. J. Am. Chem. Soc. 1961, 83, 3609.

(9) Miyata, T.; Yomosa, S. J. Phys. Soc. Jpn. 1969, 27, 727.

(10) Eisinger, J.; Shulman, R. G. Science 1968, 161, 1311.

(11) Xu, D.-G.; Nordlund, T. M. Biophys. J. 2000, 78, 1042.

(12) Douki, T.; Zalizniak, T.; Cadet, J. Photochem. Photobiol. 1997, 66, 171.

(13) Sage, E. Photochem. Photobiol 1993, 57, 163.

(14) Wilson, R. W.; Callis, P. R. J. Phys. Chem. 1976, 80, 2280.

(15) Rist, M.; Wagenknecht, H.-A.; Fiebig, T. ChemPhysChem 2002, 8, 704.

(16) Pecourt, J.-M. L.; Peon, J.; Kohler, B. J. Am. Chem. Soc. 2001, 123, 10370.

(17) Peon, J.; Zewail, A. H. Chem. Phys. Lett. 2001, 348, 255. 
(18) Onidas, D.; Markovitsi, D.; Marguet, S.; Sharonov, A.; Gustavsson, T. J. Phys. Chem. B 2002, 106, 11367.

(19) Giudice, E.; Lavery, R. Acc. Chem. Res. 2002, 35, 350.

(20) Bouvier, B.; Gustavsson, T.; Markovitsi, D.; Millié, P. Chem. Phys. 2002, 275, 75.

(21) Bouvier, B.; Dognon, J. P.; Lavery, R.; Markovitsi, D.; Millié, P.; Onidas, D.;

Zakrzewska, K. J. Phys. Chem. B 2003, 107, 13512.

(22) Frenkel, J. Phys. Rev. 1931, 37, 1276.

(23) Davydov, A. S. Theory of Molecular Excitons. In Theory of Molecular Excitons; Plenum Press: New York, 1971.

(24) Rashbah, E. I.; Sturge, M. D. Excitons; North-Holland: Amsterdam, 1982.

(25) Förster, T. Delocalization excitation and excitation transfer. In Modern Quantum Chemistry; Sinanoglu, O., Ed.; Academic Press: New York, 1965; Vol. 3; pp 93.

(26) Markovitsi, D.; Gallos, L. K.; Lemaistre, J. P.; Argyrakis, P. Chem. Phys. 2001, 269, 147. (27) Emanuele, E.; Markovitsi, D.; Millié, P.; Zakrzewska, K. ChemPhysChem 2005, in press. (28) Case, D. A.; Pearlman, D. A.; Caldwell, J. W.; Cheatham III, T. E.; Ross, W. S.; Simmerling, C. L.; Darden, T. A.; Metz, K. M.; Stanton, R. V.; Cheng, A. L.; Vincent, J. J.; Crowley, M.; Tsui, V.; Radmer, R. J.; Duan, Y.; Pitera, J.; Massova, I.; Seibel, G. L.; Singh, U. C.; Weimer, P. K.; Kollman, P. A. AMBER 6; University of California: San Francisco. 1999.

(29) Cheatham, T. E.; Cieplak, P.; Kollman, P. A. J. Biomol. Struct. Dyn. 1999, 16, 845.

(30) Berendsen, H. J. C.; Postma, J. P. M.; van Gunsteren, W. F.; DiNola, A.; Haak, J. R. J. Chem. Phys. 1984, 81, 3684.

(31) Ryckaert, J. P.; Ciccotii, G.; Berendsen, H. J. C. J. Comp. Phys. 1977, 23, 327. 
(32) Darden, T.; York, D.; Pedersen, L. J. Chem. Phys. 1993, 98, 10089.

(33) Sharp, K. A.; Honig, B. Annu. Rev. Biophys. Biophys. Chem. 1990, 19, 301.

(34) Claverie, P. Persp. Quant. Chem. Biochem. 1978, 2(Intermolecular Interactions - From Diatomics to Biopolymers), 69.

(35) Pople, J. A.; Beveridge, D. L.; Dobosh, P. A. J. Chem. Phys. 1967, 47, 2026.

(36) Dean, P. Rev. Mod. Phys. 1972, 44, 127.

(37) Schreiber, M.; Toyosawa, Y. J. Phys. Soc. Jpn. 1982, 51, 1537.

(38) Allen, F. S.; Gray, D. M.; Roberts, G. P.; Tinoco Jr., I. Biopolymers 1972, 11, 853.

(39) Huang, C.-R.; Georghiou, S. Photochem. Photobiol. 1992, 56, 95.

(40) Georghiou, S.; Zhu, S.; Weidner, R.; Huang, C.-R.; Ge, G. J. Biomol. Struct. 1990, 8, 657.

(41) Ge, G.; Georghiou, S. Photochem. Photobiol. 1991, 54, 477.

(42) Georghiou, S.; Bradrick, T. D.; Philippetis, A.; Beechem, J. Biophys. J. 1996, 70, 1909. 


\section{Figure 1}
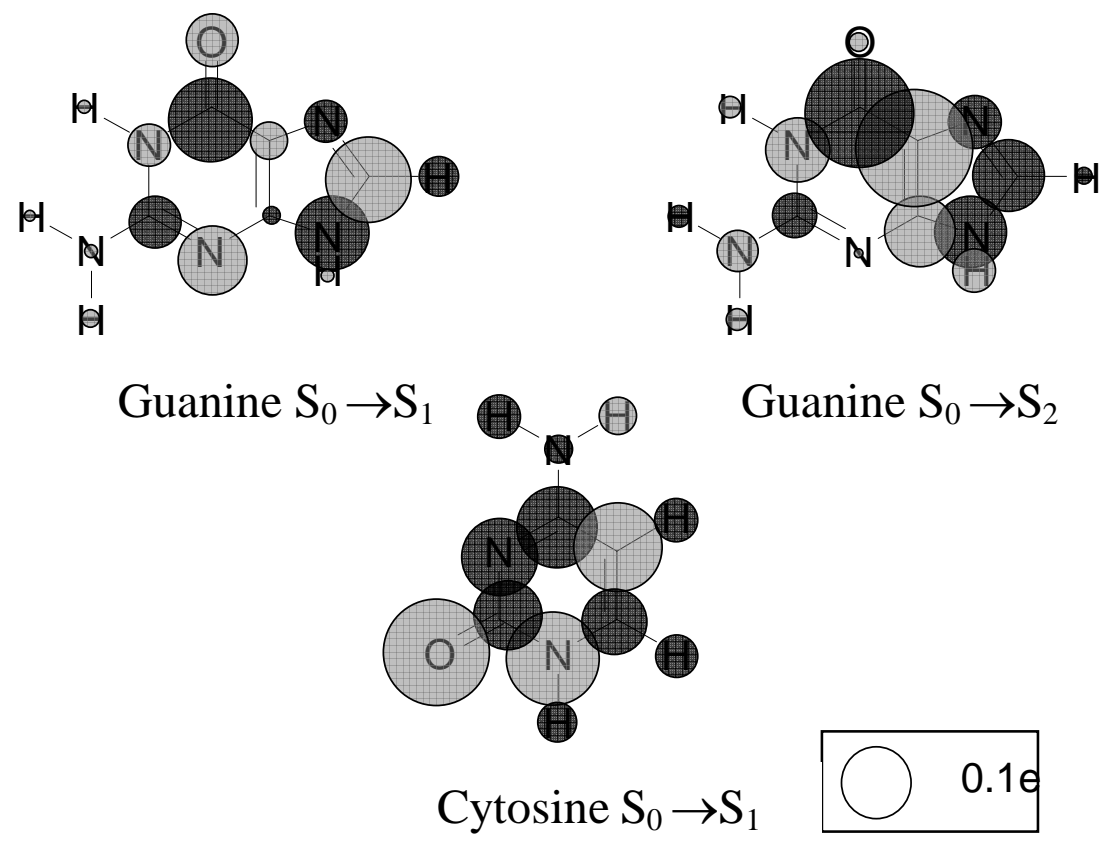
Figure 2

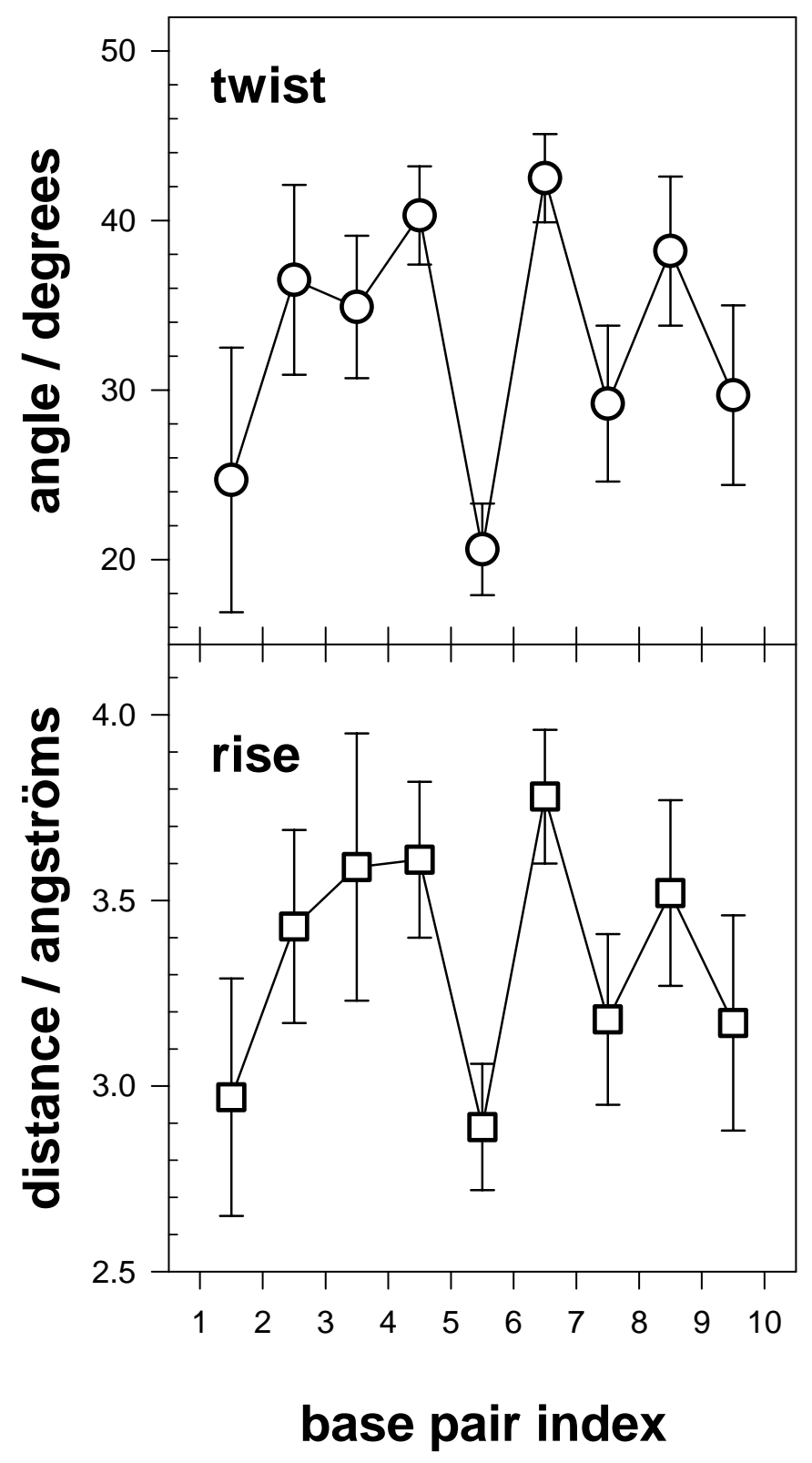


Figure 3

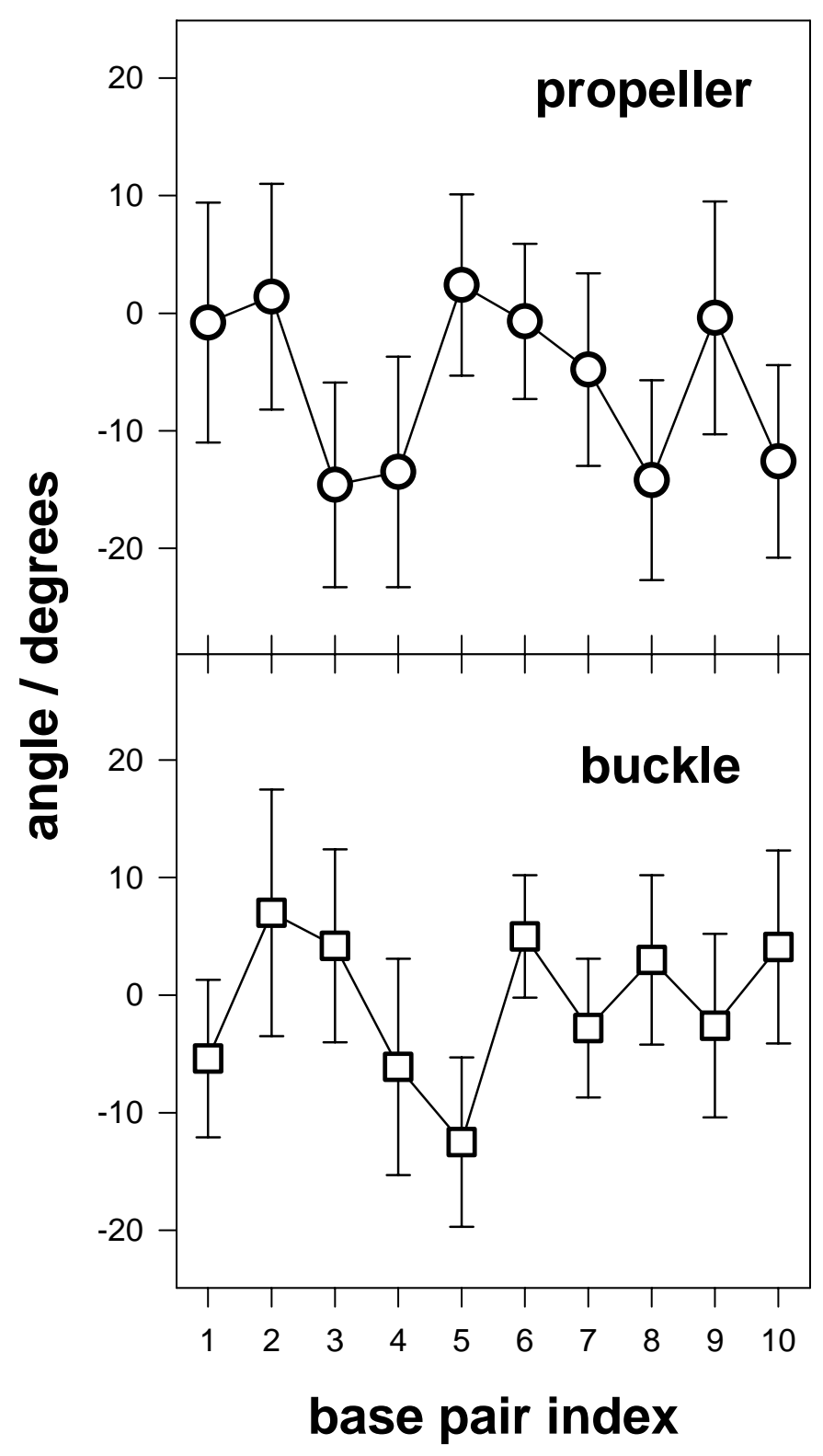




\section{Figure 4}

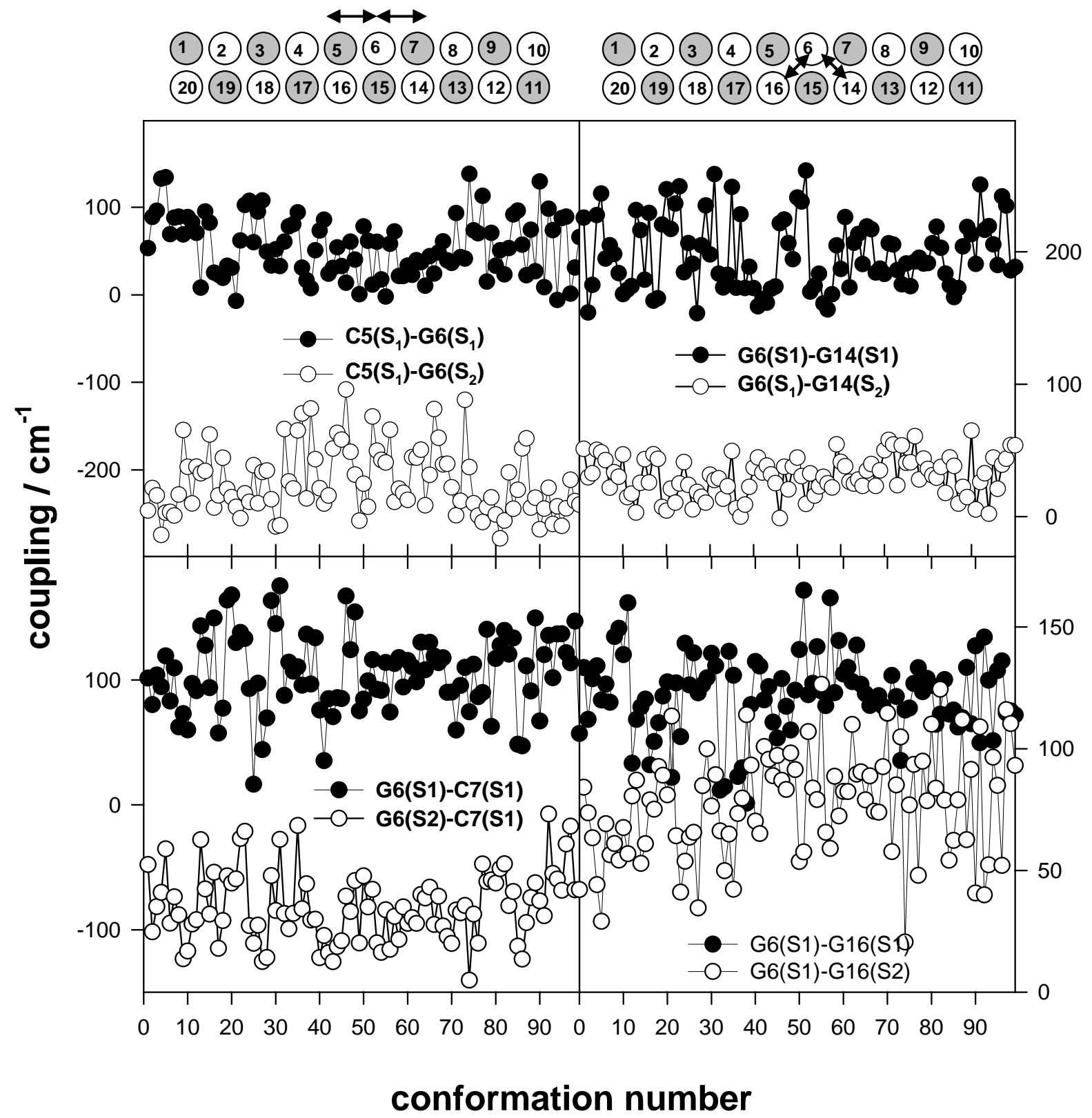


Figure 5

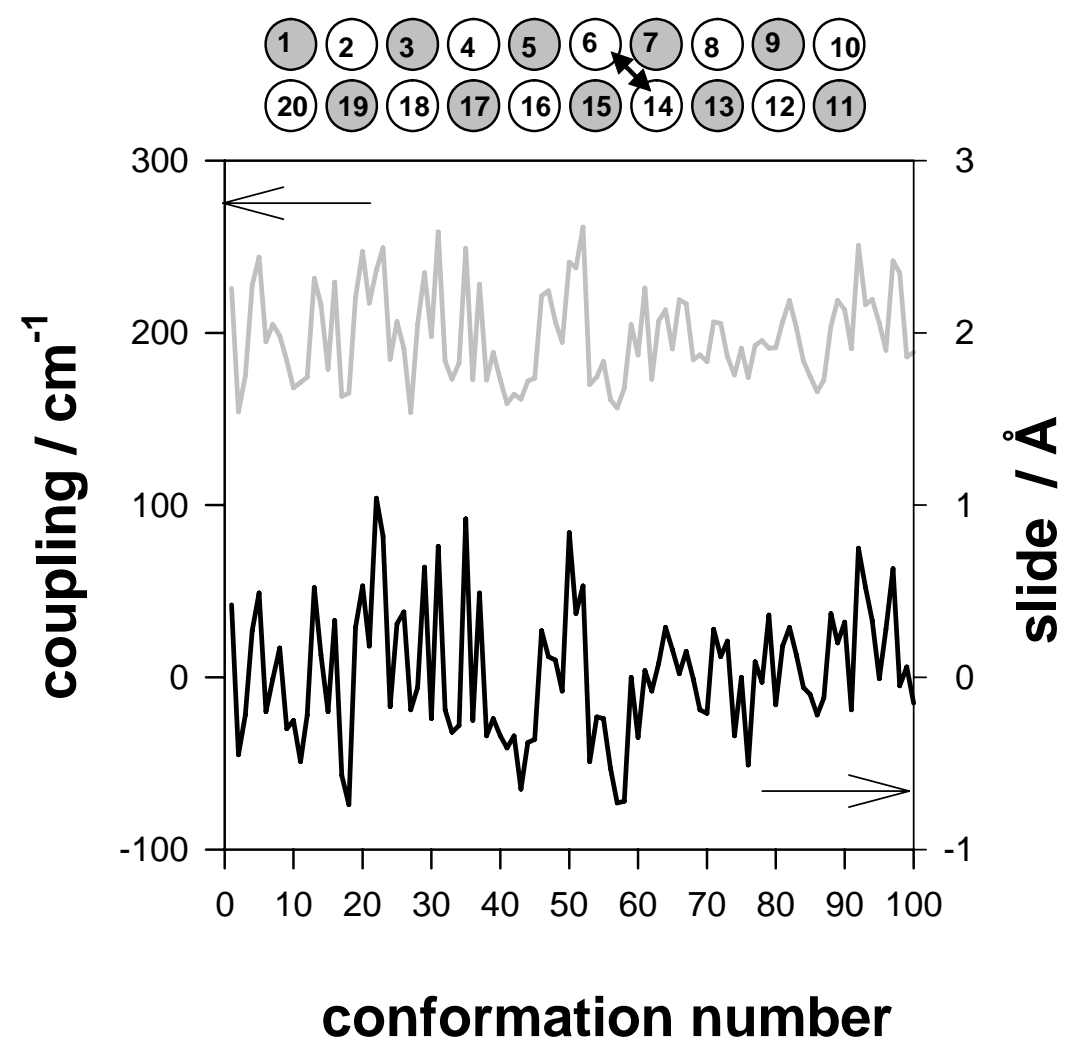


Figure 6




Figure 7

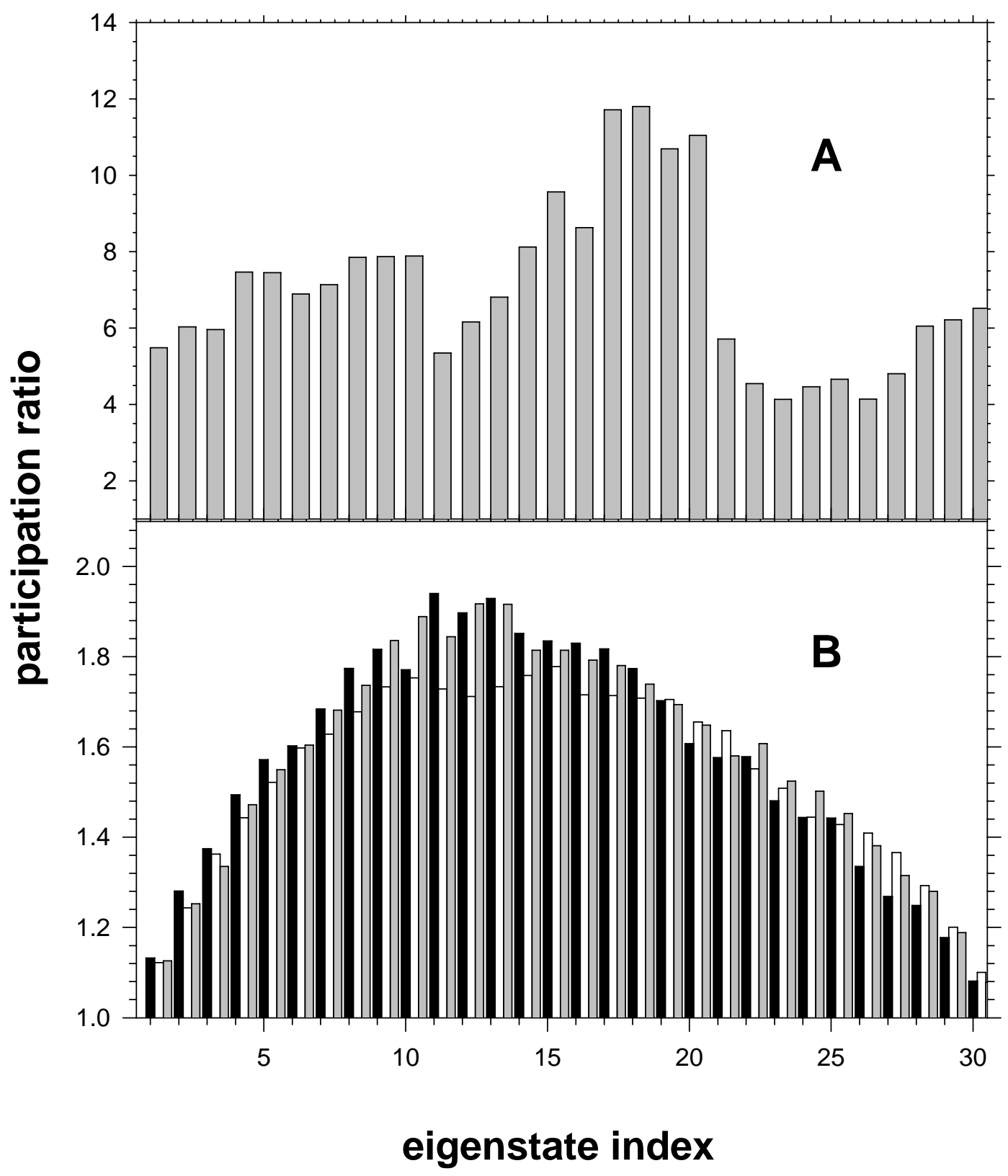


Figure 8

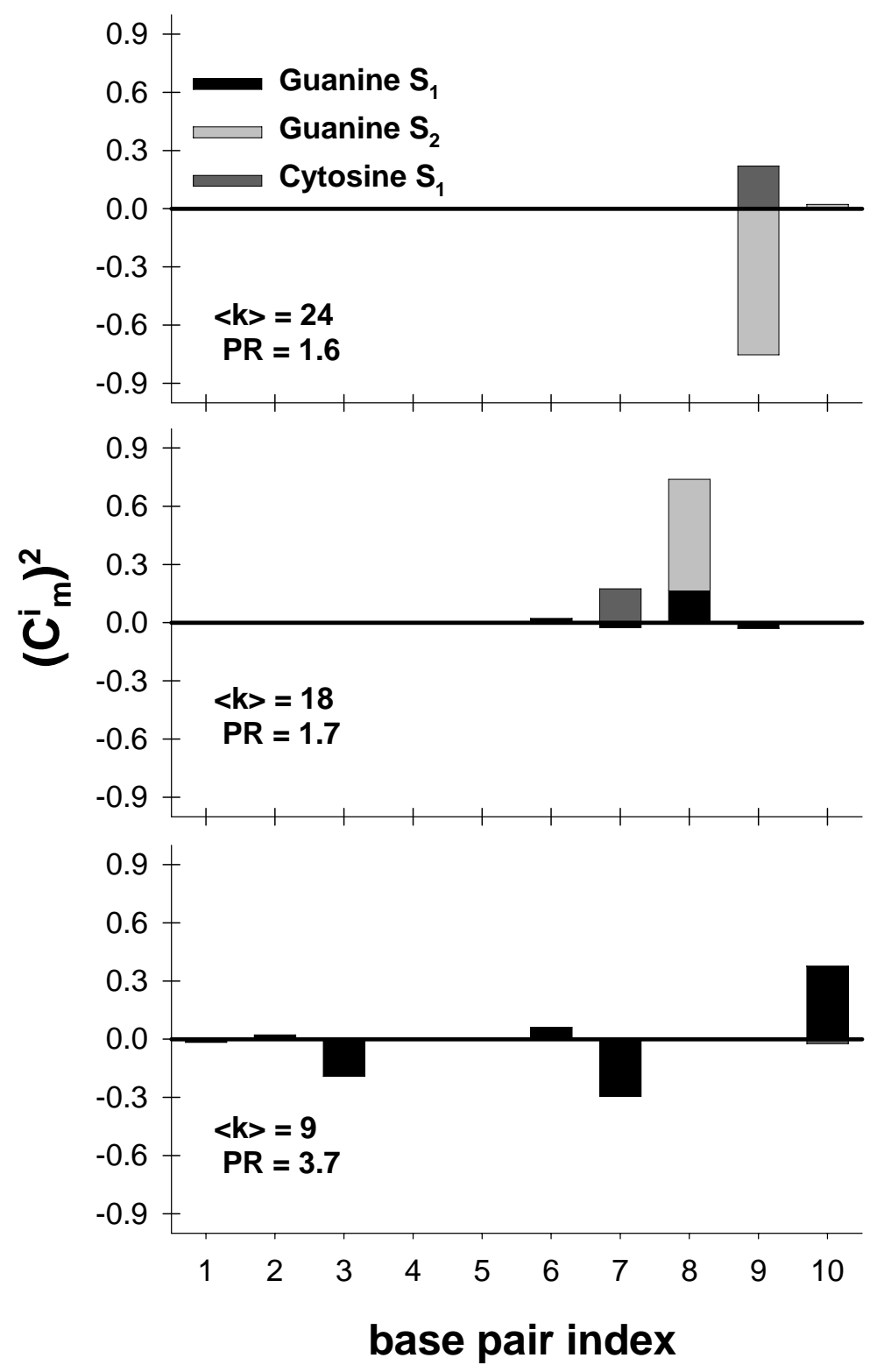




\section{Figure 9}

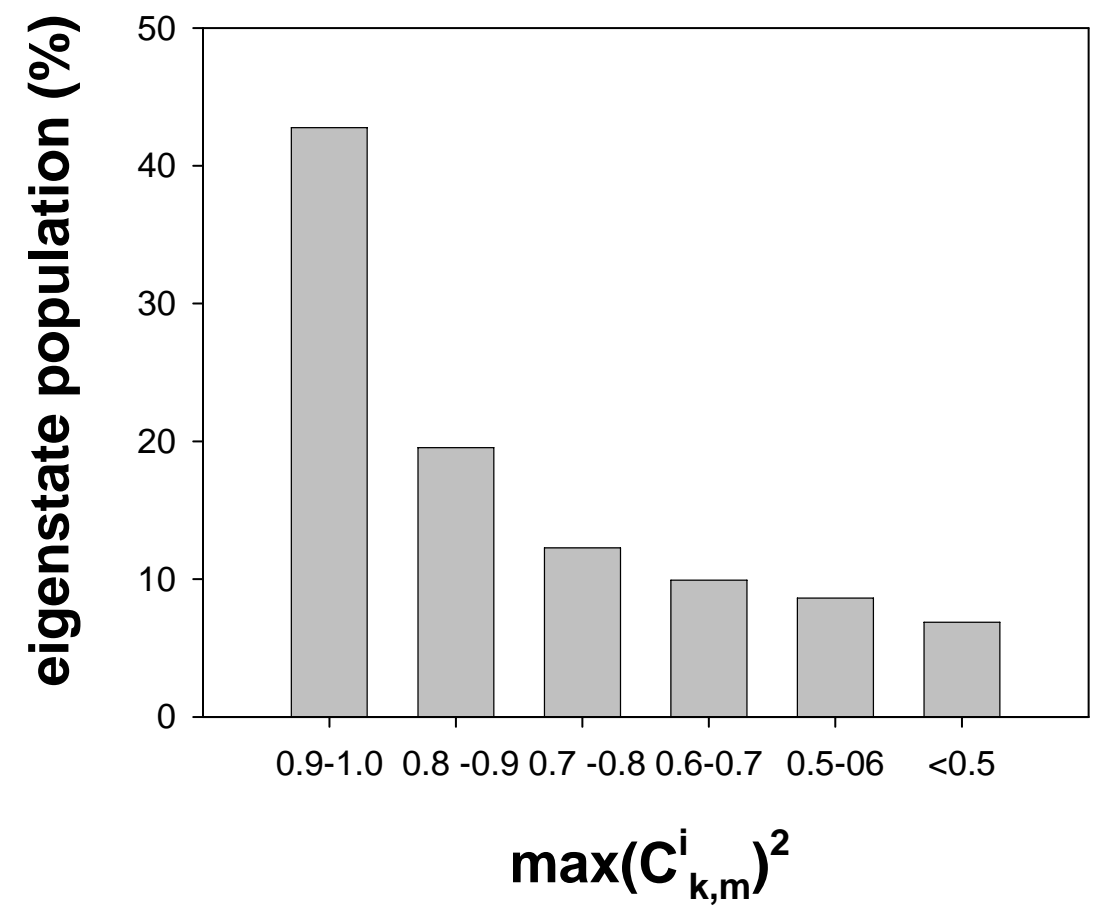


Figure 10






\section{Figure 11}

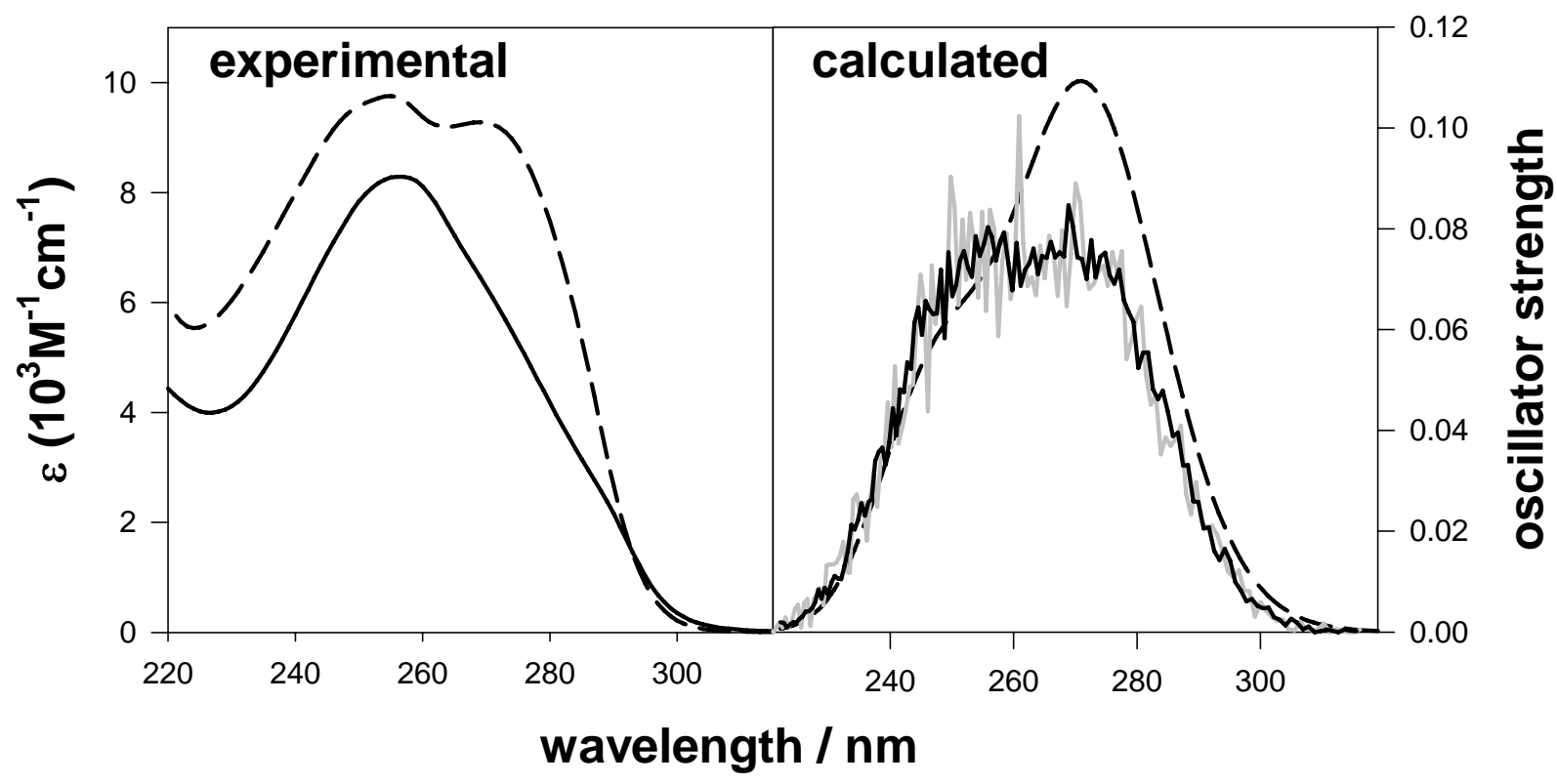




\section{Figure 12}

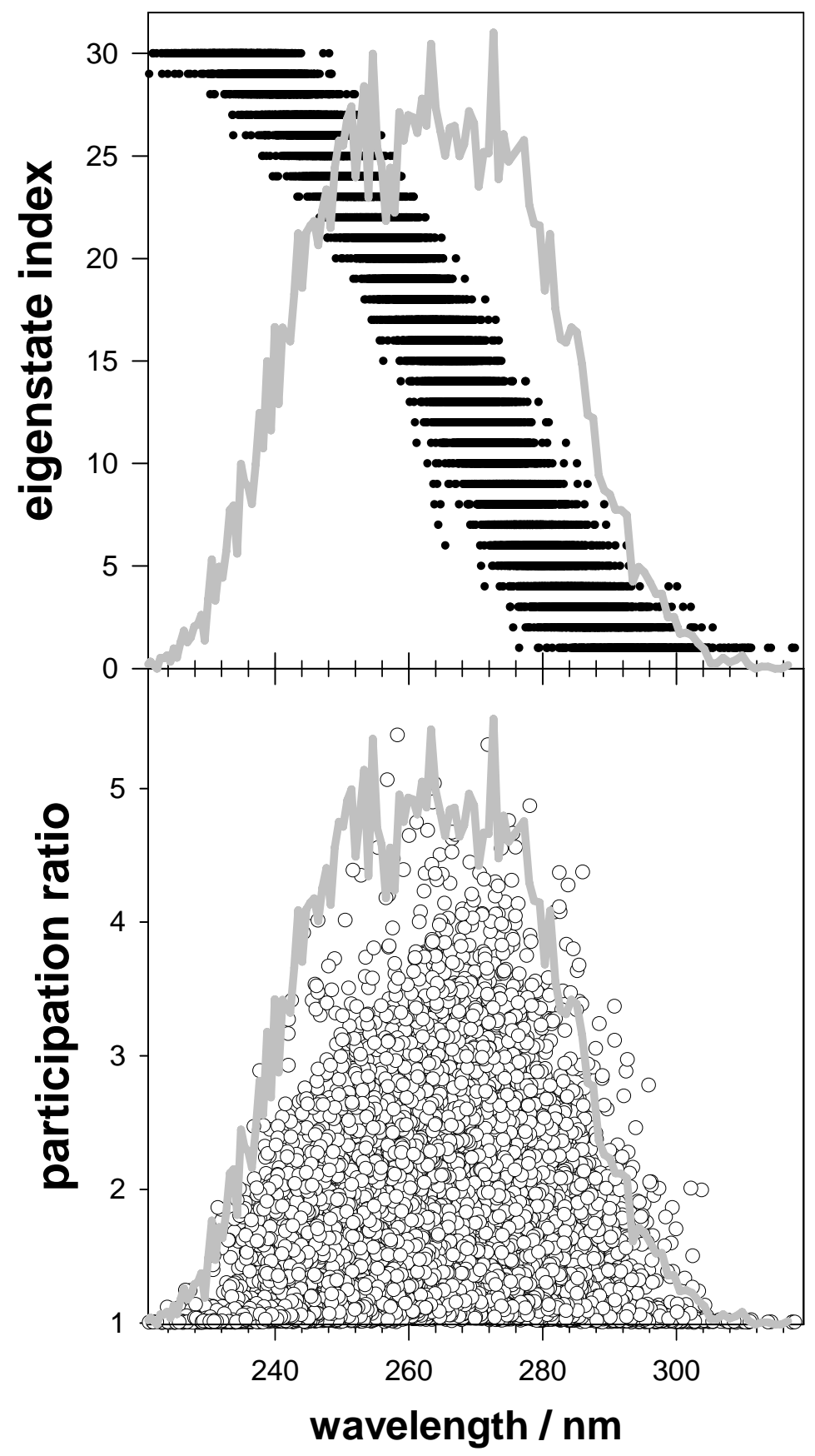

